\title{
Análisis De La Fuerza Explosiva En El Golpe De Balón Con El Pie En Los Futbolistas Categoría Senior De La Universidad Nacional De Chimborazo - Ecuador. Estudio De Caso: Test De Precisión De Golpe De Balón
}

\author{
Orlando David Mazón Moreno \\ Magister en Cultura Física y Entrenamiento Deportivo \\ Docente de la Escuela Superior Politécnica de Chimborazo \\ Jorge Giovanny Tocto Lobato \\ Magister en Cultura física y Deportología \\ Docente de la Escuela Superior Politécnica de Chimborazo \\ Ramón Fernando Bayas Machado \\ Magister en Cultura Física y Deportología \\ Docente de la Universidad Nacional de Chimborazo
}

doi: 10.19044/esj.2017.v13n14p179 URL:http://dx.doi.org/10.19044/esj.2017.v13n14p179

\begin{abstract}
The objective of the present study is to research the incidence of explosive strength on the ball kick of senior category soccer players from the "Universidad Nacional de Chimborazo". The study was carried out through the application of a comprehensive training program to improve explosive strenght and ball kick for 8 weeks. In this study, 50 soccer players were also evaluated. The age, body weight $(\mathrm{kg})$, height $(\mathrm{cm})$, and body mass index were also evaluated. The pre-post ball kick precision test was applied as well. The soccer sketch 2.5.0 program was used for the preparation of the training sessions, while the SPSS statistical package was used for the results tabulation. The results obtained are as follows: average age of 21.82, average body mass index $26.72\left(\mathrm{~kg} / \mathrm{m}^{2}\right)$. An average of 2.38 goals was obtained in the pre-test of ball precision, while an average of 3.64 goals was obtained in the post-test whit a level of significance of 0.001. In conclusion, the application of training sessions based on comprehensive exercises of explosive strength and ball kick are very important in improving the sports performance of the soccer players.
\end{abstract}

Keywords:_Explosive force, precision test, kick ball, soccer. 


\section{Resumen}

El objetivo del presente estudio fue investigar la incidencia de la fuerza explosiva en el golpe de balón con el pie, en los futbolistas categoría Senior de la Universidad Nacional de Chimborazo, mediante la aplicación de un programa de entrenamiento integrado para mejorar la fuerza explosiva y el golpe de balón con el pie, durante 8 semanas. Para el estudio fueron evaluados 50 futbolistas. Se evaluó, la edad, el peso corporal (kg), estatura (cm) y el índice de masa corporal. Así como también se aplicó el pre - post test de precisión de golpe de balón con el pie, se utilizó para la elaboración de las sesiones de entrenamiento el programa Soccer Sketch 2.5.0 y para la tabulación de los resultados el paquete estadístico SPSS. Los resultados que se obtuvieron fueron los siguientes: promedio de edad de 21,82, el promedio de peso corporal 65,80 kg, la estatura promedio 1,69 cm y el promedio del índice de masa corporal 26,72 (Kg/m2). En el pre test de precisión de golpe de balón se obtuvo una media de (2,38 goles) y en el post test una media de (3,64 goles) teniendo así un nivel de significancia del $(0,001)$. Se concluyó que la aplicación de sesiones de entrenamiento de ejercicios integrados de fuerza explosiva y golpe de balón es muy importante para mejorar el rendimiento deportivo de los futbolistas.

Palabras clave: Fuerza explosiva, test de precisión, golpe de balón, futbol

\section{INTRODUCCIÓN}

En el proceso de entrenamiento deportivo la fuerza juega un papel muy importante para alcanzar los retos propuestos y los resultandos tanto tácticos como técnicos planificados, las potencias mundiales utilizan el tiempo necesario y las tendencias más apropiadas para la preparación física, en cada deporte este proceso es distinto ya que responde a la clasificación de la actividad, el tipo de planificación utilizada, factores biomecánicos, factores fisiológicos, etc., además una excelente preparación física y el desarrollo de la fuerza, responden a cargas de entrenamiento apropiadas, que ayudan a la prevención de lesiones y a una mejor estabilidad psicológica de los deportistas.

En el Ecuador existen los clubes universitarios conformados por estudiantes activos en esta práctica deportiva, algunos con experiencia a nivel de las diferentes categorías del fútbol profesional y otros con objetivos de recreación y mantenimiento de su forma física, todos intervienen en diferentes torneos a nivel nacional, para mantener la actividad deportiva universitaria.

Los cuerpos técnicos trabajan en su planificación técnica y táctica incluyendo la preparación física pero de manera tradicional, así es como específicamente en el desarrollo de la fuerza explosiva, siendo una de las 
capacidades más importantes para el nivel del juego, no se han implementados nuevas tendencias basados en los diferentes trabajos de régimen muscular.

El poco conocimiento del entrenamiento de la fuerza explosiva por parte del entrenador, tiene como resultado, sesiones de entrenamiento empíricas para el golpe de balón, es decir, realizan los mismos trabajos o ejercicios que tienen como resultado la deserción de los deportistas de los entrenamientos de fútbol.

La inadecuada metodología de la fuerza explosiva, genera un deficiente gesto técnico del golpe de balón con el pie, en donde no toman en cuenta que el fútbol hoy en día se lo realiza de forma integral.

De acuerdo a Ozolin, (1949) menciona que la ontología de las sociedades desde sus inicios han tomado a la preparación física como algo principal dentro del entrenamiento, misma que en la actualidad se ha visto afectada por los nuevos roles que se han presentado. La preparación física ha crecido poco a poco en los últimos años en el mundo y se ha evidenciado que la preparación física es importante para el desarrollo deportivo.

Para entender de una mejor manera que es el deporte en sí, identificaremos y diferenciaremos dos términos que constantemente confundimos con la palabra deporte estos son: actividad física y ejercicio físico; actividad física es todo movimiento corporal que se produce por el sistema musculo-esquelético y realiza un gasto energético. Ejercicio físico se define como toda actividad que con acciones planificadas, estructuradas y repetitivas se adquiere un mayor gasto energético (Benhumer, Jocelyn, 2013, p. 8).

El fútbol es un deporte donde los jugadores se encuentran inmersos en una constante disputa por el balón. En el transcurso de un partido el juego se interrumpe y reanuda aproximadamente unas 120 veces. En la reanudación del juego y en todas las acciones de juego la lucha constante por la posesión del balón, este a su vez sea en ataque o en defensa, propicia que los jugadores salten, caigan, disputen, choquen, todo ello dentro de las reglas del propio juego. Para lo mismo el entrenador educador debe apoyar a sus jugadores a tratar de superar este tipo de problemas, acostumbrándolos al contacto físico con los adversarios y con el balón para tratar en menor medida que sufran caídas y tengan menor contacto con el suelo (Cabezón, Caminero, 1997).

Para practicar el deporte del fútbol como requisito fisiológico estará la fuerza; donde el deportista debe poseer una musculatura en las piernas bien desarrolladas, es decir estar apta para desarrollar una alta potencia explosiva y capaz de superar esfuerzos cortos y muy intensos (Rivas B. Milton \& Sanchez A. Erick, 2013). 
La fuerza, entendida como la capacidad para superar una resistencia, es un componente fundamental en el desarrollo de las habilidades motoras (Sánchez José, 2014, p. 33). La fuerza es una de las premisas en el desarrollo de las demás capacidades motrices, es por ello que muchos especialistas en el mundo, la llaman la capacidad madre (Rojas, Diego, Gomez Jefferson, \& Méndez Leandro, s. f., p. 1).

De acuerdo a Pérez (2013), es la capacidad de producir tensión en la musculatura al activarse, o como se entiende habitualmente al contraerse.

El autor Acdmenr (2004), dice que la fuerza es un elemento común en la vida diaria, pues cada actividad humana desde el movimiento más simple requiere de su utilización, por ello tanto en el deporte como en el trabajo cotidiano, existe un cierto tipo de producción de fuerza.

La capacidad física fuerza, es un factor determinante e importante en la mayoría de los deportes, por lo cual, si se lo desarrolla correctamente no es perjudicial en ningún caso. En la ejecución del gesto técnico golpeo de balón, juega un papel muy decisivo, que muchas veces no se debe a la falta de coordinación o habilidad, sino a la falta de conocimiento científico para el correcto desarrollo de los grupos musculares.

Los principios del entrenamiento de la fuerza analizados para esta investigación fueron la sobrecarga o también denominado principio de estímulo eficaz de la carga o de umbral. Este principio se basa en la Ley Schultz - Arnold, que nos indica que los estímulos deben superar un cierto nivel de esfuerzo, para establecer el intervalo eficaz de estimulación (Aguilar, Calahorro, \& Moral, 2009, p. 6).

La especificidad es otro de los principios que a su vez, se relaciona con la naturaleza estructural y funcional, sistemática y local de los cambios que se dan en un individuo, como resultado de un entrenamiento, estas adaptaciones son extremadamente específicas y pronosticables, ocurren sólo en el área estimulada con la sobrecarga.

Este principio es la base para el diseño del programa, es de mejor comprensión y cuando se efectúan diferentes programas de entrenamiento, a diferentes tipos de atletas, se pueden valoran los efectos logrados (Martínez, 2012, p. 4).

La fuerza explosiva puede definirse como el resultado de la relación entre la fuerza producida (manifestada o aplicada) y el tiempo necesario para ello (González Badillo, 2000; González Badillo y Ribas, 2002).

La fuerza explosiva es la capacidad de un músculo o grupo de músculos, de realizar gestos o movimientos en el menor tiempo posible, además la fuerza explosiva tiene una importante influencia en los resultados deportivos del futbol, por el corto tiempo que se dispone para poder realizar una acción técnica eficaz del golpe de balón. Los objetivos primordiales del entrenamiento de la fuerza explosiva son: el incremento de la velocidad de 
contracción, mejorar el sector neuro muscular con una ganancia máxima de coordinación del músculo y desarrollar la fuerza dinámica y la velocidad (Pérez C., 2013, p. 2).

El golpe del balón con el empeine es una de las habilidades más estudiadas en la literatura científica del fútbol, ya que se considera el gesto técnico primordial para el desarrollo de este deporte. Está generalmente aceptado que el patrón de movimiento del golpeo en fútbol, que consiste en una secuencia de movimientos de segmentos en sentido proximal - distal, ajustándose a un modelo de cadena cinética abierta (González Jurado y Pérez M, 2012, p. 1).

La presente investigación se efectúa con la selección de fútbol de la Universidad Nacional de Chimborazo, ellos cumplen una trayectoria destacada a nivel de torneos nacionales universitarios, pero no se coordinó nunca un proceso entre la investigación y la planificación deportiva de esta selección, de manera que la preparación física se ha desarrollado de manera tradicional, basada en los contenidos teóricos y prácticos básicos de esta disciplina deportiva, es por esto que se plantea implementar una nueva metódica de entrenamiento, direccionada al mejoramiento de la técnica del golpe de balón, basados en un programa planificado y sustentado, que permitirá en el desarrollo de la fuerza explosiva como el factor de mayor importancia para potenciar la técnica y el rendimiento físico de los jugadores.

\section{Metodología}

La presente investigación es de carácter cuantitativo y cualitativo.

Es cuantitativa por cuanto se utilizan procesos matemáticos (valores o datos estadísticos), que mediante la aplicación de encuestas direccionadas a los jugadores de la selección de fútbol de la UNACH, permitirá analizar los datos expuestos. Es cualitativa porque se va a valorar la fuerza explosiva y su influencia en el golpe de balón con el pie en los futbolistas categoría Senior de la UNACH.

El tema a desarrollarse comprende las dos variables de estudio que son, la variable independiente en este caso es la fuerza explosiva, y la variable dependiente como es el golpe de balón con el pie

El análisis de los datos se realizará, con el test de precisión de balón y la encuesta que permitirá hacer el análisis e interpretación de los resultados obtenidos para alcanzar los objetivos de estudio y proponer alternativas de solución encaminadas a resolver el problema detectado.

Una vez culminada la etapa de recopilación de la información, se la procesa de acuerdo a los siguientes pasos:

1. Recolección, clasificación, selección y tabulación de la información.

2. Selección de la información. 
3. Estudio estadístico de los datos.

4. Presentación de los datos en cuadros estadísticos.

5. Análisis e interpretación de los resultados

\section{Población y Muestra}

El trabajo de investigación se lo realizó, con una muestra de 50 deportistas de la categoría Senior de la UNACH. En vista, que la cantidad de personas a investigar es menor a cien, el muestreo se lo realizó con el cien por ciento de los deportistas.

Tabla Nº1. Población y Muestra

\begin{tabular}{|c|c|c|}
\hline Población & Muestra & Porcentaje \\
\hline Deportistas & 50 & $100 \%$ \\
\hline Total & 50 & $100 \%$ \\
\hline
\end{tabular}

Elaborado por: Lic. Orlando David Mazón Moreno

\section{RESULTADOS:}

Análisis del test de precisión del golpe de balón con el pie antes de la aplicación del programa de entrenamiento integrado de fuerza a los futbolistas de la selección de la UNACH

Tabla $N^{\circ} 2$ Datos generales del test de precisión del balón con el pie (Pre Test)

\begin{tabular}{|c|c|c|c|c|c|c|c|c|}
\hline$N^{\circ}$ & POSICIÓN & EDAD & $\begin{array}{c}\text { PESO } \\
\text { EN KG }\end{array}$ & TALLA & IMC & CLASIFICACIÓN & $\begin{array}{c}\text { PRE } \\
\text { TEST } \\
\text { GOLES }\end{array}$ & CALIFICACIÓN \\
\hline 1 & Arquero & 20 & 68 & 1,68 & 28,44 & Sobrepeso & 3 & Bueno \\
\hline 2 & Arquero & 22 & 67 & 1,67 & 28,45 & Sobrepeso & 1 & Malo \\
\hline 3 & Arquero & 21 & 69 & 1,69 & 28,43 & Sobrepeso & 2 & Regular \\
\hline 4 & Arquero & 22 & 65 & 1,7 & 26,37 & Sobrepeso & 3 & Bueno \\
\hline 5 & Arquero & 23 & 69 & 1,75 & 25,91 & Sobrepeso & 3 & Bueno \\
\hline 6 & Defensa & 24 & 68 & 1,65 & 29,76 & Sobrepeso & 2 & Regular \\
\hline 7 & Defensa & 25 & 62 & 1,73 & 24,02 & Normal & 2 & Regular \\
\hline 8 & Defensa & 23 & 65 & 1,68 & 27,19 & Sobrepeso & 4 & Muy Bueno \\
\hline 9 & Defensa & 22 & 65 & 1,65 & 28,45 & Sobrepeso & 3 & Bueno \\
\hline 10 & Defensa & 21 & 65 & 1,67 & 27,6 & Sobrepeso & 3 & Bueno \\
\hline 11 & Defensa & 20 & 63 & 1,7 & 25,56 & Sobrepeso & 3 & Bueno \\
\hline 12 & Defensa & 20 & 64 & 1,67 & 27,18 & Sobrepeso & 2 & Regular \\
\hline 13 & Defensa & 23 & 65 & 1,69 & 26,78 & Sobrepeso & 2 & Regular \\
\hline 14 & Defensa & 24 & 68 & 1,72 & 26,75 & Sobrepeso & 1 & Malo \\
\hline 15 & Defensa & 22 & 70 & 1,72 & 27,54 & Sobrepeso & 2 & Regular \\
\hline 16 & Defensa & 21 & 65 & 1,68 & 27,19 & Sobrepeso & 3 & Bueno \\
\hline 17 & Defensa & 25 & 62 & 1,72 & 24,39 & Normal & 2 & Regular \\
\hline 18 & Defensa & 25 & 70 & 1,71 & 27,97 & Sobrepeso & 3 & Bueno \\
\hline 19 & Defensa & 23 & 63 & 1,72 & 24,79 & Normal & 2 & Regular \\
\hline 20 & Defensa & 22 & 65 & 1,65 & 28,45 & Sobrepeso & 2 & Regular \\
\hline 21 & Defensa & 21 & 70 & 1,68 & 29,28 & Sobrepeso & 3 & Bueno \\
\hline 22 & Volante & 20 & 62 & 1,69 & 25,54 & Sobrepeso & 3 & Bueno \\
\hline
\end{tabular}




\begin{tabular}{|c|c|c|c|c|c|c|c|c|}
\hline 23 & Volante & 19 & 61 & 1,73 & 23,63 & Normal & 3 & Bueno \\
\hline 24 & Volante & 19 & 65 & 1,75 & 24,41 & Normal & 2 & Regular \\
\hline 25 & Volante & 22 & 68 & 1,68 & 28,44 & Sobrepeso & 3 & Bueno \\
\hline 26 & Volante & 21 & 70 & 1,75 & 26,29 & Sobrepeso & 3 & Bueno \\
\hline 27 & Volante & 24 & 66 & 1,67 & 28,03 & Sobrepeso & 2 & Regular \\
\hline 28 & Volante & 24 & 64 & 1,65 & 28,01 & Sobrepeso & 2 & Regular \\
\hline 29 & Volante & 22 & 63 & 1,68 & 26,35 & Sobrepeso & 3 & Bueno \\
\hline 30 & Volante & 21 & 62 & 1,72 & 24,39 & Normal & 2 & Regular \\
\hline 31 & Volante & 20 & 65 & 1,73 & 25,18 & Sobrepeso & 3 & Bueno \\
\hline 32 & Volante & 19 & 63 & 1,66 & 27,16 & Sobrepeso & 3 & Bueno \\
\hline 33 & Volante & 19 & 67 & 1,68 & 28,03 & Sobrepeso & 1 & Malo \\
\hline 34 & Volante & 20 & 70 & 1,7 & 28,4 & Sobrepeso & 1 & Malo \\
\hline 35 & Volante & 20 & 71 & 1,72 & 27,94 & Sobrepeso & 2 & Regular \\
\hline 36 & Volante & 23 & 63 & 1,69 & 25,95 & Sobrepeso & 3 & Bueno \\
\hline 37 & Volante & 25 & 62 & 1,63 & 27,96 & Sobrepeso & 3 & Bueno \\
\hline 38 & Volante & 22 & 66 & 1,68 & 27,61 & Sobrepeso & 2 & Regular \\
\hline 39 & Volante & 24 & 66 & 1,73 & 25,57 & Sobrepeso & 2 & Regular \\
\hline 40 & Volante & 22 & 68 & 1,75 & 25,54 & Sobrepeso & 2 & Regular \\
\hline 41 & Volante & 21 & 70 & 1,73 & 27,12 & Sobrepeso & 3 & Bueno \\
\hline 42 & Volante & 20 & 62 & 1,69 & 25,54 & Sobrepeso & 2 & Regular \\
\hline 43 & Delantero & 19 & 61 & 1,68 & 25,52 & Sobrepeso & 3 & Bueno \\
\hline 44 & Delantero & 23 & 65 & 1,74 & 24,79 & Normal & 3 & Bueno \\
\hline 45 & Delantero & 24 & 67 & 1,73 & 25,96 & Sobrepeso & 1 & Malo \\
\hline 46 & Delantero & 25 & 70 & 1,69 & 28,84 & Sobrepeso & 2 & Regular \\
\hline 47 & Delantero & 23 & 71 & 1,73 & 27,51 & Sobrepeso & 2 & Regular \\
\hline 48 & Delantero & 22 & 64 & 1,72 & 25,18 & Sobrepeso & 3 & Bueno \\
\hline 49 & Delantero & 19 & 67 & 1,68 & 28,03 & Sobrepeso & 2 & Regular \\
\hline 50 & Delantero & 20 & 63 & 1,68 & 26,35 & Sobrepeso & 2 & Regular \\
\hline
\end{tabular}

Fuente: Datos de los deportistas de la Categoría Senior de la Universidad Nacional de

Chimborazo

Elaborado por: Equipo de trabajo

Tabla No 3 Estadísticos descriptivos del test de Precisión del Golpe de Balón con el pie

\begin{tabular}{|c|c|}
\hline & Pre test \\
\hline n. & 50 \\
\hline Media & 2,38 \\
\hline Desv. Tip &, 69 \\
\hline Mínimo & 1 \\
\hline Máximo & 4 \\
\hline
\end{tabular}

Fuente: Datos de los deportistas de la Categoría Senior de la Universidad Nacional de Chimborazo

Elaborado por: Lic. Orlando David Mazón Moreno

Los deportistas de la selección de la UNACH en su pre test de precisión de golpe de balón no fueron muy efectivos, para lo cual se debe fortalecer, mediante ejercicios integrados tanto en la parte física y técnica para mejorar la precisión de los futbolistas.

Tabla No 4 Frecuencia del test de Precisión de Golpe de Balón con el pie (Pre Test) 


\begin{tabular}{|c|c|c|c|}
\hline & \multicolumn{3}{|c|}{ Pre Test } \\
\cline { 2 - 4 } & Goles & Media & Frecuencia. \\
\hline Malo & 1 & 5 & 10 \\
\hline Regular & 2 & 22 & 44 \\
\hline Bueno & 3 & 22 & 44 \\
\hline Muy Bueno & 4 & 1 & 2 \\
\hline Excelente & 5 & - & - \\
\hline Total & & 50 & 100 \\
\hline
\end{tabular}

Fuente: Datos de los deportistas de la Categoría Senior de la Universidad Nacional de Chimborazo

Elaborado por: Lic. Orlando David Mazón Moreno

Los jugadores se encuentran en el rango de regular y bueno, teniendo en cuenta que el golpe de balón de precisión con el pie es muy importante al momento de realizar un pase en un encuentro de fútbol, para lo cual en las sesiones de entrenamiento trataremos de mejorar este gesto técnico fundamental.

Programa de entrenamiento integrado para mejorar la fuerza explosiva y el golpe de balón con el pie en los futbolistas categoría senior de la Universidad Nacional de Chimborazo

\section{Test de precisión de golpe de balón}

Elementos: 5 vallas, 5 balones. Descripción: Un jugador trata de introducir los balones en las pequeñas porterías (vallas). Se contabilizan los aciertos.

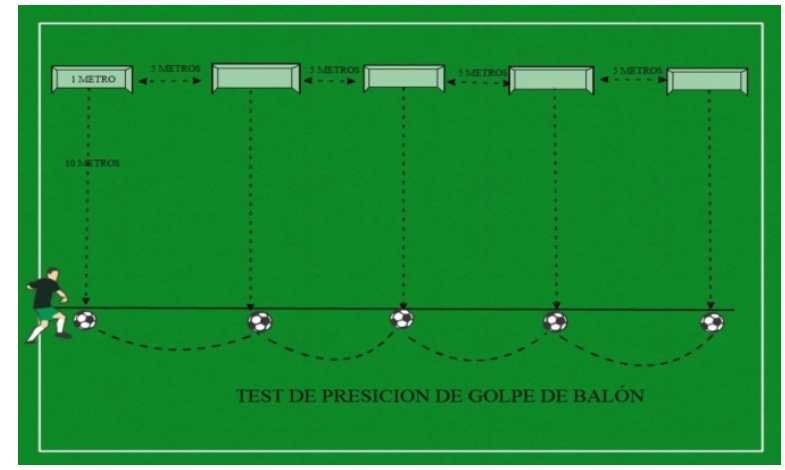

Gráfico $\mathbf{N}^{\mathbf{0}} \mathbf{1}$. Test de precisión.

Elaborado por: Lic. Orlando David Mazón Moreno 
Tabla $N^{0} 5$ Valoración de referencia del golpe de precisión de balon con el pie

\begin{tabular}{|c|c|}
\hline Goles & Calificación \\
\hline 1 & Malo \\
\hline 2 & Regular \\
\hline 3 & Bueno \\
\hline 4 & Muy Bueno \\
\hline 5 & Excelente \\
\hline
\end{tabular}

Fuente: Lic. Orlando David Mazón Moreno

Elaborado por: Lic. Orlando David Mazón Moreno

A continuación se detallan 2 de las 21 sesiones del Programa de Entrenamiento Integrado de Fuerza Explosiva, realizados para mejorar la fuerza integral, para desarrollar la fuerza explosiva y el golpe de balón mediante un circuito integrado aplicado a los futbolistas de la categoría Senior de la UNACH.

Tabla $\mathrm{N}^{\circ} 6$ Sesión de Entrenamiento $\mathrm{N}^{\circ} 1$

\begin{tabular}{|c|c|c|c|c|c|}
\hline \multicolumn{6}{|c|}{ SESIÓN DE ENTRENAMIENTO No 1} \\
\hline \multicolumn{2}{|c|}{ Institución: UNACH } & \multicolumn{2}{|c|}{$\begin{array}{l}\text { Entrenador: Lcdo. David Mazón } \\
\text { Moreno }\end{array}$} & \multicolumn{2}{|c|}{ Fecha: 26-10-2015 } \\
\hline \multicolumn{2}{|c|}{ Deporte: Fútbol } & \multicolumn{2}{|c|}{ Tiempo de duración: 100 minutos } & \multicolumn{2}{|c|}{ Micro ciclo: 1} \\
\hline \multicolumn{2}{|c|}{ Categoría: Senior } & \multicolumn{2}{|l|}{ Género: Masculino } & \multicolumn{2}{|c|}{ Escenario: Gimnasio } \\
\hline \multicolumn{6}{|c|}{ Objetivo: Desarrollar la fuerza general a los deportistas de la categoría Senior de la UNACH. } \\
\hline $\mathbf{N}^{\mathbf{o}}$ & ACTIVIDADES & DOSCIFICACION & TIEMPO & $\begin{array}{c}\text { MEDIO } \\
\text { METODO }\end{array}$ & RECURSOS \\
\hline P.I. & $\begin{array}{l}>\text { Motivación } \\
>\text { Calentamiento general } \\
\text { segmentos corporales } \\
>\text { Calentamiento especifico } \\
>\text { Estiramiento }\end{array}$ & $\begin{array}{l}2 \\
3 \\
8 \\
2\end{array}$ & $15^{\prime}$ & $\begin{array}{l}\text { Explicativo } \\
\text { Demostrativo } \\
\text { Series } \\
\text { Repeticiones }\end{array}$ & $\begin{array}{l}\text { Sala de } \\
\text { Gimnasio } \\
\text { Conos } \\
\text { Cinta métrica } \\
\text { Silbato } \\
\text { Cronómetro } \\
\text { Balones }\end{array}$ \\
\hline P.P & $\begin{array}{l}\text { Trabajo de fuerza al } \mathbf{3 0 \%} \\
\text { Sentadilla Profunda } \\
\text { Press de banco } \\
\text { Extensiones } \\
\text { Velocidad de distancia de } \\
\text { 10m. } \\
\text { Fútbol en espacio reducido }\end{array}$ & $\begin{array}{l}3(15 \text { rep x } 2 \text { pausa })= \\
15^{`} \\
3(15 \text { rep x } 2 \text { ’pausa })= \\
15^{`} \\
3(15 \text { rep x } 2 \text { pausa })= \\
15^{`} \\
\frac{3\left(5 \text { rep } \times 30^{`}\right)}{2 `}=10^{`} \\
2\left(10^{`}\right)=20^{`}\end{array}$ & $75^{`}$ & & \\
\hline $\begin{array}{l}\text { P.F } \\
\cdot\end{array}$ & $\begin{array}{l}\text { VUELTA A LA } \\
\text { CALMA } \\
\text { (Trote suave e ir bajando } \\
\text { la intensidad) } \\
\text { ESTIRAMIENTO }\end{array}$ & $\begin{array}{l}1 \text { ( } 5 \text { minutos trote ) a } \\
100 \text { a } 110 \text { pulsaciones }\end{array}$ & 10 & & \\
\hline
\end{tabular}

Elaborado por: Lic. Orlando David Mazón Moreno 
Tabla $\mathrm{N}^{\mathrm{0}} 7$ Sesión de Entrenamiento $\mathrm{N}^{\mathrm{0}} 21$

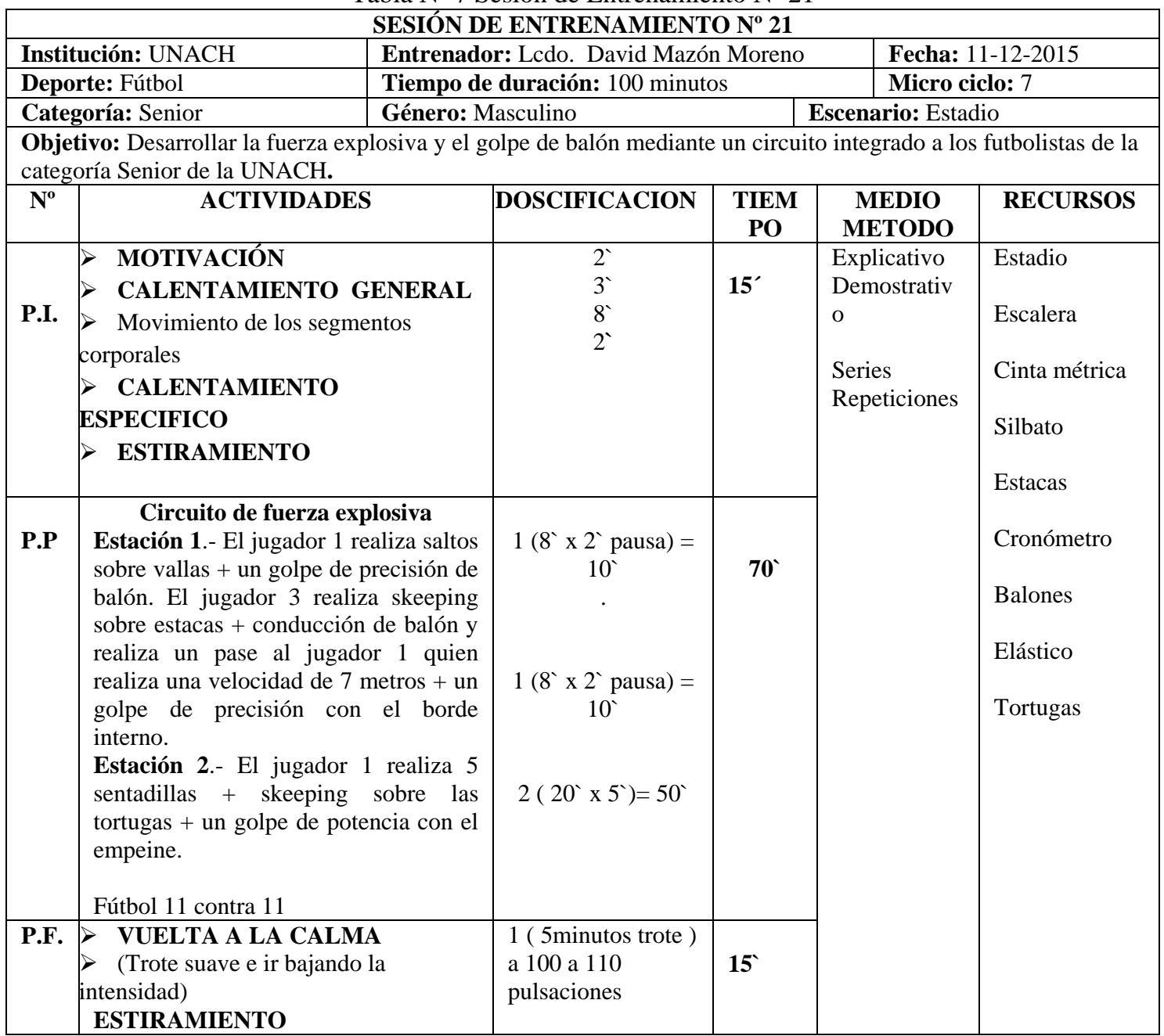

Elaborado por: Lic. Orlando David Mazón Moreno

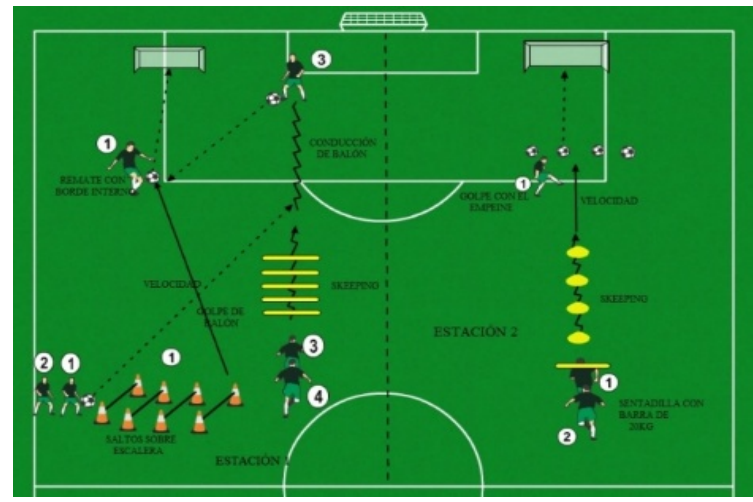

Gráfico $\mathbf{N}^{\mathbf{0}} 2$ Circuito $\mathrm{N}^{\circ} 15$

Elaborado por: Lic. Orlando David Mazón Moreno 


\section{Análisis del test de precisión del golpe de balón con el pie después de la aplicación del programa de entrenamiento integrado de fuerza a los futbolistas de la selección de la UNACH}

Tabla No 8 Datos generales del Post Test de Precisión de Golpe de Balón con el pie

\begin{tabular}{|c|c|c|c|c|c|c|c|c|}
\hline $\mathbf{N}^{\mathbf{o}}$ & POSICIÓN & EDAD & PESO EN KG & TALLA & IMC & CLASIFICACIÓN & $\begin{array}{c}\text { POST } \\
\text { TEST } \\
\text { GOLES }\end{array}$ & CALIFICACIÓN \\
\hline & Arquero & 20 & 68 & 1,68 & 28,44 & Sobrepeso & 2 & Regular \\
\hline 2 & Arquero & 22 & 67 & 1,67 & 28,45 & Sobrepeso & 3 & Bueno \\
\hline 3 & Arquero & 21 & 69 & 1,69 & 28,43 & Sobrepeso & 4 & Muy Bueno \\
\hline 4 & Arquero & 22 & 65 & 1,7 & 26,37 & Sobrepeso & 4 & Muy Bueno \\
\hline 5 & Arquero & 23 & 69 & 1,75 & 25,91 & Sobrepeso & 4 & Muy Bueno \\
\hline 6 & Defensa & 24 & 68 & 1,65 & 29,76 & Sobrepeso & 4 & Muy Bueno \\
\hline 7 & Defensa & 25 & 62 & 1,73 & 24,02 & Normal & 3 & Bueno \\
\hline 8 & Defensa & 23 & 65 & 1,68 & 27,19 & Sobrepeso & 2 & Regular \\
\hline 9 & Defensa & 22 & 65 & 1,65 & 28,45 & Sobrepeso & 5 & Excelente \\
\hline 10 & Defensa & 21 & 65 & 1,67 & 27,6 & Sobrepeso & 5 & Excelente \\
\hline 11 & Defensa & 20 & 63 & 1,7 & 25,56 & Sobrepeso & 4 & Muy Bueno \\
\hline 12 & Defensa & 20 & 64 & 1,67 & 27,18 & Sobrepeso & 3 & Bueno \\
\hline 13 & Defensa & 23 & 65 & 1,69 & 26,78 & Sobrepeso & 4 & Muy Bueno \\
\hline 14 & Defensa & 24 & 68 & 1,72 & 26,75 & Sobrepeso & 3 & Bueno \\
\hline 15 & Defensa & 22 & 70 & 1,72 & 27,54 & Sobrepeso & 4 & Muy Bueno \\
\hline 16 & Defensa & 21 & 65 & 1,68 & 27,19 & Sobrepeso & 4 & Muy Bueno \\
\hline 17 & Defensa & 25 & 62 & 1,72 & 24,39 & Normal & 4 & Muy Bueno \\
\hline 18 & Defensa & 25 & 70 & 1,71 & 27,97 & Sobrepeso & 5 & Excelente \\
\hline 19 & Defensa & 23 & 63 & 1,72 & 24,79 & Normal & 4 & Muy Bueno \\
\hline 20 & Defensa & 22 & 65 & 1,65 & 28,45 & Sobrepeso & 4 & Muy Bueno \\
\hline 21 & Defensa & 21 & 70 & 1,68 & 29,28 & Sobrepeso & 3 & Bueno \\
\hline 22 & Volante & 20 & 62 & 1,69 & 25,54 & Sobrepeso & 4 & Muy Bueno \\
\hline 23 & Volante & 19 & 61 & 1,73 & 23,63 & Normal & 3 & Bueno \\
\hline 24 & Volante & 19 & 65 & 1,75 & 24,41 & Normal & 3 & Bueno \\
\hline 25 & Volante & 22 & 68 & 1,68 & 28,44 & Sobrepeso & 3 & Bueno \\
\hline 26 & Volante & 21 & 70 & 1,75 & 26,29 & Sobrepeso & 4 & Muy Bueno \\
\hline 27 & Volante & 24 & 66 & 1,67 & 28,03 & Sobrepeso & 4 & Muy Bueno \\
\hline 28 & Volante & 24 & 64 & 1,65 & 28,01 & Sobrepeso & 3 & Bueno \\
\hline 29 & Volante & 22 & 63 & 1,68 & 26,35 & Sobrepeso & 4 & Muy Bueno \\
\hline 30 & Volante & 21 & 62 & 1,72 & 24,39 & Normal & 4 & Muy Bueno \\
\hline 31 & Volante & 20 & 65 & 1,73 & 25,18 & Sobrepeso & 3 & Bueno \\
\hline 32 & Volante & 19 & 63 & 1,66 & 27,16 & Sobrepeso & 2 & Regular \\
\hline 33 & Volante & 19 & 67 & 1,68 & 28,03 & Sobrepeso & 4 & Muy Bueno \\
\hline 34 & Volante & 20 & 70 & 1,7 & 28,4 & Sobrepeso & 3 & Bueno \\
\hline 35 & Volante & 20 & 71 & 1,72 & 27,94 & Sobrepeso & 4 & Muy Bueno \\
\hline 36 & Volante & 23 & 63 & 1,69 & 25,95 & Sobrepeso & 4 & Muy Bueno \\
\hline 37 & Volante & 25 & 62 & 1,63 & 27,96 & Sobrepeso & 4 & Muy Bueno \\
\hline 38 & Volante & 22 & 66 & 1,68 & 27,61 & Sobrepeso & 3 & Bueno \\
\hline 39 & Volante & 24 & 66 & 1,73 & 25,57 & Sobrepeso & 4 & Muy Bueno \\
\hline 40 & Volante & 22 & 68 & 1,75 & 25,54 & Sobrepeso & 3 & Bueno \\
\hline 41 & Volante & 21 & 70 & 1,73 & 27,12 & Sobrepeso & 3 & Bueno \\
\hline 42 & Volante & 20 & 62 & 1,69 & 25,54 & Sobrepeso & 4 & Muy Bueno \\
\hline 43 & Delantero & 19 & 61 & 1,68 & 25,52 & Sobrepeso & 5 & Excelente \\
\hline 44 & Delantero & 23 & 65 & 1,74 & 24,79 & Normal & 3 & Bueno \\
\hline 45 & Delantero & 24 & 67 & 1,73 & 25,96 & Sobrepeso & 3 & Bueno \\
\hline 46 & Delantero & 25 & 70 & 1,69 & 28,84 & Sobrepeso & 4 & Muy Bueno \\
\hline
\end{tabular}




\begin{tabular}{|l|l|l|l|l|l|l|l|l|}
\hline 47 & Delantero & 23 & 71 & 1,73 & 27,51 & Sobrepeso & 4 & Muy Bueno \\
\hline 48 & Delantero & 22 & 64 & 1,72 & 25,18 & Sobrepeso & 4 & Muy Bueno \\
\hline 49 & Delantero & 19 & 67 & 1,68 & 28,03 & Sobrepeso & 4 & Muy Bueno \\
\hline 50 & Delantero & 20 & 63 & 1,68 & 26,35 & Sobrepeso & 4 & Muy Bueno \\
\hline
\end{tabular}

Fuente: Datos de los deportistas de la Categoría Senior de la Universidad Nacional de Chimborazo

Elaborado por: Equipo de investigadores

Tabla No 6 Estadísticos descriptivos Golpe de Balón con el pie (Post Test)

\begin{tabular}{|c|c|}
\hline & Post test \\
\hline n. & 50 \\
\hline Media & 3,64 \\
\hline Desv. Tip &, 72 \\
\hline Mínimo & 2 \\
\hline Máximo & 5 \\
\hline
\end{tabular}

Fuente: Datos de los deportistas de la Categoría Senior de la Universidad Nacional de Chimborazo

Elaborado por: Lic. Orlando David Mazón Moreno.

Los deportistas de la selección de la UNACH en su pos test de precisión de golpe de balón con el pie fueron más efectivos, en donde mediante ejercicios integrados en la parte física y técnica se logró mejorar la precisión del golpe de balón con el pie, realizando golpes de balón en distancias de 5, 8, 10 metros con el borde interno y con el empeine.

Tabla N 9 Frecuencia del test de Precisión de Golpe de Balón con el pie (Post Test)

\begin{tabular}{|l|c|c|c|}
\hline \multirow{2}{*}{} & \multicolumn{3}{|c|}{ Post Test } \\
\cline { 2 - 4 } & Goles & Media & Frecuencia. \\
\hline Malo & 1 & - & - \\
\hline Regular & 2 & 3 & 7 \\
\hline Bueno & 3 & 16 & 31 \\
\hline Muy Bueno & 4 & 27 & 53 \\
\hline Excelente & 5 & 4 & 9 \\
\hline Total & & 50 & 100 \\
\hline
\end{tabular}

Fuente: Datos de los deportistas de la Categoría Senior de la Universidad Nacional de Chimborazo

Elaborado por: Lic. Orlando David Mazón Moreno

Luego de aplicar los correctos ejercicios mediante una planificación específica encontramos a los jugadores en su mayoría en rango de muy bueno y excelente, esto quiere decir que los ejercicios lograron mejorar este gesto técnico muy efectivo al momento de realizar un pase. 
ANALISIS DE LA RELACIÓN ENTRE EL PRE TES Y POST TEST DE PRECISIÒN DEL GOLPE DE BALÒN CON EL PIE EN LOS FUTBOLISTAS DE LA SELECCIÒN DE LA UNACH

Tabla No 10 Datos Generales del Pre y Post Test de Precisión del Golpe de Balón con el pie

\begin{tabular}{|c|c|c|c|c|c|c|c|c|c|c|}
\hline NÚMERO & POSICIÓN & EDAD & PESO EN KG & TALLA & IMC & CLASIFICACIÓN & $\begin{array}{c}\text { PRE } \\
\text { TEST } \\
\text { GOLES }\end{array}$ & CALIFICACIÓN & $\begin{array}{l}\text { POST TEST } \\
\text { GOLES }\end{array}$ & CALIFICACIÓN \\
\hline 1 & Arquero & 20 & 68 & 1,68 & 28,44 & Sobrepeso & 3 & Bueno & 2 & Regular \\
\hline 2 & Arquero & 22 & 67 & 1,67 & 28,45 & Sobrepeso & 1 & Malo & 3 & Bueno \\
\hline 3 & Arquero & 21 & 69 & 1,69 & 28,43 & Sobrepeso & 2 & Regular & 4 & Muy Bueno \\
\hline 4 & Arquero & 22 & 65 & 1,7 & 26,37 & Sobrepeso & 3 & Bueno & 4 & Muy Bueno \\
\hline 5 & Arquero & 23 & 69 & 1,75 & 25,91 & Sobrepeso & 3 & Bueno & 4 & Muy Bueno \\
\hline 6 & Defensa & 24 & 68 & 1,65 & 29,76 & Sobrepeso & 2 & Regular & 4 & Muy Bueno \\
\hline 7 & Defensa & 25 & 62 & 1,73 & 24,02 & Normal & 2 & Regular & 3 & Bueno \\
\hline 8 & Defensa & 23 & 65 & 1,68 & 27,19 & Sobrepeso & 4 & Muy Bueno & 2 & Regular \\
\hline 9 & Defensa & 22 & 65 & 1,65 & 28,45 & Sobrepeso & 3 & Bueno & 5 & Excelente \\
\hline 10 & Defensa & 21 & 65 & 1,67 & 27,6 & Sobrepeso & 3 & Bueno & 5 & Excelente \\
\hline 11 & Defensa & 20 & 63 & 1,7 & 25,56 & Sobrepeso & 3 & Bueno & 4 & Muy Bueno \\
\hline 12 & Defensa & 20 & 64 & 1,67 & 27,18 & Sobrepeso & 2 & Regular & 3 & Bueno \\
\hline 13 & Defensa & 23 & 65 & 1,69 & 26,78 & Sobrepeso & 2 & Regular & 4 & Muy Bueno \\
\hline 14 & Defensa & 24 & 68 & 1,72 & 26,75 & Sobrepeso & 1 & Malo & 3 & Bueno \\
\hline 15 & Defensa & 22 & 70 & 1,72 & 27,54 & Sobrepeso & 2 & Regular & 4 & Muy Bueno \\
\hline 16 & Defensa & 21 & 65 & 1,68 & 27,19 & Sobrepeso & 3 & Bueno & 4 & Muy Bueno \\
\hline 17 & Defensa & 25 & 62 & 1,72 & 24,39 & Normal & 2 & Regular & 4 & Muy Bueno \\
\hline 18 & Defensa & 25 & 70 & 1,71 & 27,97 & Sobrepeso & 3 & Bueno & 5 & Excelente \\
\hline 19 & Defensa & 23 & 63 & 1,72 & 24,79 & Normal & 2 & Regular & 4 & Muy Bueno \\
\hline 20 & Defensa & 22 & 65 & 1,65 & 28,45 & Sobrepeso & 2 & Regular & 4 & Muy Bueno \\
\hline 21 & Defensa & 21 & 70 & 1,68 & 29,28 & Sobrepeso & 3 & Bueno & 3 & Bueno \\
\hline 22 & Volante & 20 & 62 & 1,69 & 25,54 & Sobrepeso & 3 & Bueno & 4 & Muy Bueno \\
\hline 23 & Volante & 19 & 61 & 1,73 & 23,63 & Normal & 3 & Bueno & 3 & Bueno \\
\hline 24 & Volante & 19 & 65 & 1,75 & 24,41 & Normal & 2 & Regular & 3 & Bueno \\
\hline 25 & Volante & 22 & 68 & 1,68 & 28,44 & Sobrepeso & 3 & Bueno & 3 & Bueno \\
\hline 26 & Volante & 21 & 70 & 1,75 & 26,29 & Sobrepeso & 3 & Bueno & 4 & Muy Bueno \\
\hline 27 & Volante & 24 & 66 & 1,67 & 28,03 & Sobrepeso & 2 & Regular & 4 & Muy Bueno \\
\hline
\end{tabular}




\begin{tabular}{|c|c|c|c|c|c|c|c|c|c|c|}
\hline 28 & Volante & 24 & 64 & 1,65 & 28,01 & Sobrepeso & 2 & Regular & 3 & Bueno \\
\hline 29 & Volante & 22 & 63 & 1,68 & 26,35 & Sobrepeso & 3 & Bueno & 4 & Muy Bueno \\
\hline 30 & Volante & 21 & 62 & 1,72 & 24,39 & Normal & 2 & Regular & 4 & Muy Bueno \\
\hline 31 & Volante & 20 & 65 & 1,73 & 25,18 & Sobrepeso & 3 & Bueno & 3 & Bueno \\
\hline 32 & Volante & 19 & 63 & 1,66 & 27,16 & Sobrepeso & 3 & Bueno & 2 & Regular \\
\hline 33 & Volante & 19 & 67 & 1,68 & 28,03 & Sobrepeso & 1 & Malo & 4 & Muy Bueno \\
\hline 34 & Volante & 20 & 70 & 1,7 & 28,4 & Sobrepeso & 1 & Malo & 3 & Bueno \\
\hline 35 & Volante & 20 & 71 & 1,72 & 27,94 & Sobrepeso & 2 & Regular & 4 & Muy Bueno \\
\hline 36 & Volante & 23 & 63 & 1,69 & 25,95 & Sobrepeso & 3 & Bueno & 4 & Muy Bueno \\
\hline 37 & Volante & 25 & 62 & 1,63 & 27,96 & Sobrepeso & 3 & Bueno & 4 & Muy Bueno \\
\hline 38 & Volante & 22 & 66 & 1,68 & 27,61 & Sobrepeso & 2 & Regular & 3 & Bueno \\
\hline 39 & Volante & 24 & 66 & 1,73 & 25,57 & Sobrepeso & 2 & Regular & 4 & Muy Bueno \\
\hline 40 & Volante & 22 & 68 & 1,75 & 25,54 & Sobrepeso & 2 & Regular & 3 & Bueno \\
\hline 41 & Volante & 21 & 70 & 1,73 & 27,12 & Sobrepeso & 3 & Bueno & 3 & Bueno \\
\hline 42 & Volante & 20 & 62 & 1,69 & 25,54 & Sobrepeso & 2 & Regular & 4 & Muy Bueno \\
\hline 43 & Delantero & 19 & 61 & 1,68 & 25,52 & Sobrepeso & 3 & Bueno & 5 & Excelente \\
\hline 44 & Delantero & 23 & 65 & 1,74 & 24,79 & Normal & 3 & Bueno & 3 & Bueno \\
\hline 45 & Delantero & 24 & 67 & 1,73 & 25,96 & Sobrepeso & 1 & Malo & 3 & Bueno \\
\hline 46 & Delantero & 25 & 70 & 1,69 & 28,84 & Sobrepeso & 2 & Regular & 4 & Muy Bueno \\
\hline 47 & Delantero & 23 & 71 & 1,73 & 27,51 & Sobrepeso & 2 & Regular & 4 & Muy Bueno \\
\hline 48 & Delantero & 22 & 64 & 1,72 & 25,18 & Sobrepeso & 3 & Bueno & 4 & Muy Bueno \\
\hline 49 & Delantero & 19 & 67 & 1,68 & 28,03 & Sobrepeso & 2 & Regular & 4 & Muy Bueno \\
\hline 50 & Delantero & 20 & 63 & 1,68 & 26,35 & Sobrepeso & 2 & Regular & 4 & Muy Bueno \\
\hline
\end{tabular}

Fuente: Datos de los deportistas de la Categoría Senior de la Universidad Nacional de Chimborazo

Elaborado por: Lic. Orlando David Mazón Moreno 
Tabla No 11 Estadísticos del Pre y Post Test de la Precisión del golpe de Balón con el Pie

\begin{tabular}{|c|c|c|c|c|}
\hline & $\mathbf{n}$. & Media & Dev. Tip. & $\mathbf{x}^{2}$ \\
\hline Pre Test & 50 & 2,38 &, 69 & .001 \\
\hline Post Test & 50 & 3,64 &, 72 & \\
\hline
\end{tabular}

Fuente: Datos de los deportistas de la Categoría Senior de la Universidad Nacional de Chimborazo

Elaborado por: Lic. Orlando David Mazón Moreno

Se obtuvo resultados significativos en la media y la desviación típica, en el gesto técnico golpe de precisión con el pie, mediante el programa de entrenamiento integrado, utilizando ejercicios específicos con balón sin descuidar la parte física y táctica en los deportistas de la selección de la UNACH.

Tabla N 12 Frecuencia de la Precisión del golpe de Balón con el Pie (Pre Test y Post Test)

\begin{tabular}{|l|c|c|c|c|c|}
\hline \multirow{2}{*}{} & & \multicolumn{2}{|c|}{ Pre Test } & \multicolumn{2}{c|}{ Post Test } \\
\cline { 2 - 6 } & Goles & Media & Frecuencia. & Media & Frecuencia. \\
\hline Malo & 1 & 5 & 10,00 & - & - \\
\hline Regular & 2 & 22 & 44,00 & 3 & 7,00 \\
\hline Bueno & 3 & 22 & 44,00 & 16 & 31,00 \\
\hline Muy Bueno & 4 & 1 & 2,00 & 27 & 53,00 \\
\hline Excelente & 5 & - & - & 4 & 9,00 \\
\hline Total & & 50 & 100 & 50 & 100 \\
\hline
\end{tabular}

Fuente: Datos de los deportistas de la Categoría Senior de la Universidad Nacional de Chimborazo

Elaborado por: Lic. Orlando David Mazón Moreno

Luego de aplicar ejercicios específicos de golpe de precisión de balón con el pie, se obtuvieron resultados muy satisfactorios, si realizamos un análisis podemos observar que en el pre test existían 5 jugadores que realizaban 1 gol, mientras que en el post test existen 4 jugadores que realizan 5 goles.

\section{Conclusion}

La implementación del programa de entrenamiento integrado de fuerza explosiva ha sido muy beneficioso ya que en este programa se utilizó una nueva metodología de ejercicios de forma dinámica, estática - dinámica, ejercicios en circuitos, utilizando una correcta y adecuada planificación deportiva.

En cuanto al universo de estudio, los deportistas de la categoría Senior de la Universidad Nacional de Chimborazo, se logró analizar el golpe de balón mediante la utilización del test de precisión, obteniendo resultados positivos para esta investigación. La media en el pre test del golpe de balón fue de 2,38 goles mientras que en el post test fue de 3,64 goles.

El programa de entrenamiento integrado que fue dirigido a los futbolistas de la categoría Senior de la Universidad Nacional de Chimborazo, 
ha sido efectuado con mucho éxito, para lo cual las sesiones de entrenamiento se han planificado y dosificado de acuerdo a las condiciones físicas de los futbolistas. Por lo tanto este programa se lo ha llevado a cabo con normalidad, donde a los deportistas se les controlaba la frecuencia cardiaca y se los proporcionaba el adecuado descanso para la realización de los ejercicios

\section{References:}

1. Aguilar, J., Calahorro, F., \& Moral. J. (2009). A condición física y el entrenamiento: objetivos y principios. FEAFYS, 12.

2. Acdmenr, A. (2004). Conceptos básicos sobre la fuerza muscular. Recuperado 15 de septiembre de 2015, a partir de http://www.efdeportes.com/efd190/conceptos-basicos-sobre-lafuerza-muscular.htm

3. Benhumer, J. (2013). Deporte que se relaciona con mayor flexibilidad de columna lumbar en niños de 10 a 12 años que practican fútbol, natación y basquetbol en la escuela del deporte de Toluca «Javier García Moreno Requénez» del ciclo escolar 2012 2013. Universidad Autónoma Del Estado De México, México.

4. Cabezón Y., y Caminero F. (1997). Fútbol. Una propuesta curricular a través del juego. Unidades didácticas para Secundaria VII. INDE.

5. González Jurado, y Pérez M. (2012). Diferencias en parámetros cinemáticos del golpeo en fútbol entre hombres y mujeres, 12.

6. Martínez, P. (2012). El entrenamiento de fuerza.

7. Pérez C. (2013). Metodología y valoración del entrenamiento de la fuerza.

8. Rivas B. Milton, \& Sánchez A. Erick. (2013). Entrenamiento actual de la condición física del futbolista. MHSalud, 10, 1-131.

9. Rojas Diego, Gómez Jefferson, \& Méndez Leandro. (s.f.). Caracterización de la fuerza explosiva en futbolistas de la categoría sub 15 de Villavicencio. Impetus, 14.

10. Sánchez, J. (2014). La fuerza aplicada al tenis de campo y su incidencia en el golpe de derecha listado en los jugadores de la categoría sub 14 y sub 16 en la escuela de tenis del club Los Arrayanes en la ciudad de Quito, provincia de Pichincha. Universidad Técnica de Ambato. Ambato. 